\title{
Evaluación de una aplicación web con participación pública en la planificación espacial y toma de decisiones para la integración de construcciones rurales
}

\author{
An assessment of web application with public participation in spatial \\ planning and decision-making for rural building integrations
}

\author{
J. S. Jeong ${ }^{(*)}$, L. García-Moruno ${ }^{(*)}$, J. Hernández-Blanco ${ }^{(* *)}$, M. J. Montero-Parejo ${ }^{(* *)}$
}

\section{RESUMEN}

Se presenta una aplicación web que facilita la integración de construcciones turísticas rurales en su entorno mediante la participación ciudadana. El objetivo general ha sido examinar cómo la investigación puede contribuir a apoyar la toma de decisiones de los usuarios, así como su aplicación a un caso de estudio empírico en Hervás, España. A partir de los datos obtenidos de los participantes, agrupados en cuatro grupos diferentes, este estudio examinó la identificación de modelos espaciales para las distintas percepciones y el intercambio de conocimientos de los procesos de integración de construcciones en el entorno rural, la certificación del posible impacto en el turismo y la definición de una interfaz de usabilidad. Los resultados demostraron que esta web puede facilitar un consenso sobre las recomendaciones para la ordenación del territorio con la implementación de alternativas de decisión.

Palabras clave: Web participativa; participación ciudadana; planificación colaborativa espacial; integración de construcciones rurales; toma de decisiones; percepción de los usuarios.

\section{ABSTRACT}

This paper presents the final web implementation and statistical survey results from a continuing broad research project. In this work, a web spatial planning application with public participation was developed to support suitable rural tourism building integrations into a landscape. The general goal of this paper is to examine how research can contribute to support users' decision-making, together with its application to an empirical case study in Hervás, Spain. With the participants' data set grouped by four different clusters, this study examined the identification of spatial models for the different perceptions and knowledge sharing of building integrations into a rural landscape, the certification of the possible impact on tourism and the definition of interface usability. The results demonstrated the web could achieve consensus on recommendations for the spatial planning with the implementation of decision alternatives.

Keywords: Participatory web; public participation; collaborative spatial planning; rural building integration; decision-making; user perceptions.

${ }^{(*)}$ Centro Universitario de Mérida - Universidad de Extremadura. Mérida (España).

(**) Centro Universitario de Plasencia - Universidad de Extremadura. Plasencia (España).

Persona de contacto/Corresponding author: jin@unex.es (J. S. Jeong).

Cómo citar este artículo/Citation: Jeong, J. S., García-Moruno, L., Hernández-Blanco, J., Montero-Parejo, M. J. (2015). Evaluación de una aplicación web con participación pública en la planificación espacial y toma de decisiones para la integración de construcciones rurales. Informes de la Construcción, 67(539): eo98, doi: http://dx.doi.org/10.3989/ic.14.035.

Licencia / License: Salvo indicación contraria, todos los contenidos de la edición electrónica de Informes de la Construcción se distribuyen bajo una licencia de uso y distribución Creative Commons Reconocimiento no Comercial 3.o. España (cc-by-nc). 


\section{INTRODUCCIÓN}

Durante las últimas décadas, en el sur de Europa, se pueden encontrar muchos casos donde se ha producido una relación discordante entre edificios rurales y sus integraciones en el paisaje (1) (2) (3) (4). Por otro lado, el turismo ha sido identificado como una herramienta poderosa para el desarrollo, mediante el estímulo del crecimiento económico a través del aumento de las divisas, la inversión de los pequeños propietarios y el incremento del empleo local. En algunos casos, esto se ha traducido en una mayor protección del entorno y el aumento de la financiación para los proyectos de conservación del medio ambiente (5) (6). La importancia del turismo rural también ha sido reconocida por la Organización Mundial del Turismo a través del análisis de su situación actual y perspectivas (7) (8) (9). La política de planificación del paisaje europea toma medidas para proteger su identidad cultural y para promover la calidad de paisaje a través de los códigos de construcción (10). Sin embargo, la integración adecuada de las construcciones rurales en un paisaje, sigue sin ser una consideración general en los procesos de planificación (11) (12) (13) (14), donde, los profesionales deberían tener en cuenta la integración adecuada de las edificaciones, con objeto de mantener y/o mejorar un equilibrio armonioso con el entorno del paisaje (15) (16).

En muchas organizaciones, los participantes encuentran oportunidades para lograr sus objetivos individuales a través de la acción colectiva (17) (18). La participación ciudadana resulta efectiva en organizaciones de diferentes tamaños, desde las más pequeñas dedicadas a la búsqueda de soluciones a los problemas locales, a las más grandes que pueden resolver problemas más sofisticados de alcance regional. En particular, los agentes decisores tienen que prestar atención a la participación pública y de las organizaciones de la comunidad, debido a la importancia que tiene el aporte de la comunidad a la definición de los intereses locales (19). Desde el punto de vista de la ordenación del territorio, la gran mayoría de las partes involucradas poseen diferentes orígenes, intereses, grados de autoridad en el proceso de toma de decisiones o distintas interpretaciones de los problemas, y deben organizarse para alcanzar soluciones factibles consensuadas por la comunidad, ya que las soluciones así alcanzadas tienden a ser razonables, realistas y sostenibles (20). La modelización espacial permite el análisis de grandes volúmenes de datos espaciales a través de un sistema de información geográfica (SIG), ya que permite dar una expresión geográfica de las políticas económicas, sociales, culturales y ecológicas (21) (22) (23) (24) (25). Por lo tanto, un proceso de colaboración es la manera correcta con la que la conciliación de los enfoques individuales y la toma de decisiones puede satisfacer a la mayoría o a todos los participantes (1) (26).

El desarrollo de las técnicas computacionales e internet, principalmente en las últimas décadas, ha venido acompañado de diferentes esfuerzos para implementar herramientas integradoras, capaces de hacer frente tanto a los aspectos analíticos como comunicativos de la ordenación del territorio dentro de un marco único (27) (28) (26) (29) (30). Algunos investigadores han mencionado que internet ofrece una nueva manera de facilitar los procesos para la toma de decisiones participativas y generar una nuevo espacio público que apoye la interacción y el debate entre los participantes (31) (32) (33). Los agentes decisores están desarrollando actualmente procedimientos para trabajar de forma eficaz y eficiente con los participantes a través del uso de estas tecnologías, para ga- rantizar oportunidades a las partes interesadas mediante la colaboración asíncrona y distribuida (34) (35). Por lo tanto, el análisis de decisión multi-criterio (ADMC), con el apoyo de la tecnología existente, puede proporcionar a los responsables de tomas de decisiones, mecanismos de ayuda para explorar y resolver múltiples problemas en la toma de decisiones complicadas (36) (37) (38) (39) (40). Uno de los métodos de toma de decisiones, ampliamente aceptado, es el proceso analítico jerárquico (PAJ). Este procedimiento constituye un enfoque eficaz porque puede ser utilizado para generar decisiones alternativas en una situación específica de toma de decisiones (41) (42). Por lo general, los criterios tomados en el PAJ tienen diferentes significados, lo que refleja la diversidad de preferencia de los participantes con respecto a cada criterio (43).

En esta investigación se describe la implementación de una página web de planificación participativa, en la que se incluyen un conjunto de métodos capaces de identificar y formular criterios adecuados y establecer modelos espaciales para la correcta integración de la ordenación del territorio, con el objetivo principal de resaltar las interrelaciones existentes entre las edificaciones turísticas rurales y sus entornos. La aplicación web implementada permite a los usuarios calibrar sus métodos, medir su percepción y habilidades de intercambio de conocimientos en lo que respecta a la integración de construcciones, definir cómo de adecuada han encontrado la interfaz web y su posible impacto en el turismo.

\section{MATERIALES Y MÉTODO}

La región de estudio, Hervás $\left(60 \mathrm{~km}^{2}\right)$, se sitúa en el centrooeste de España, y se caracteriza por poseer muchas zonas de alto valor biológico, paisajístico y recreativo, tales como ríos y humedales, consideradas atracciones turísticas durante la temporada de verano. Esto ha motivado un rápido crecimiento de los desarrollos rurales, y como consecuencia se ha producido una pérdida de los paisajes tradicionales, así como un cambio sustancial del uso de la tierra, aunque la definición de lo que constituye una zona rural puede diferir entre países, e incluso regiones dentro de un país (2). Concretamente, se ha producido un importante incremento en el desarrollo de edificios dedicados al turismo rural debido al crecimiento de la demanda de residencias de vacaciones o de las características naturales de la zona (44) (45). A pesar de la existencia de una ley regional extremeña (LESOTEX, Ley 15/2001 del suelo y ordenación territorial de Extremadura), aún no se dan respuestas coherentes a los problemas de la especificación de uso de la tierra o de la ordenación del territorio rural (46). Por esta razón, con esta investigación se sugiere un enfoque para establecer la localización de edificaciones de turismo rural dentro de un paisaje, teniendo en cuenta criterios amplios y una evaluación por etapas. Para ello, se ha aplicado un proceso de evaluación multidisciplinar, enmarcado en una interfaz web, a través de las herramientas de análisis espacial con ADMC, basándose en ciertos criterios de evaluación (físicos, ambientales y socio-económicos) y restricciones. Como último paso los datos obtenidos, a través de cuestionarios de los usuarios de esta herramienta web han sido estudiados y estadísticamente analizados.

\subsection{Aplicación de tomas de decisiones mediante análisis multi-criterio}

Se han considerado de forma extensiva los criterios y procesos de evaluación, y posteriormente se han clasificado en seis 
restricciones y tres criterios principales, cada uno con cuatro sub-criterios que intervienen en el proceso de cálculo de la siguiente manera (2):

\section{Criterios de evaluación:}

Factores relativos a evaluaciones físicas: para el área de estudio seleccionada se analizaron los siguientes cuatro factores; (A) morfología: ya que tiene un papel importante para la derivación de atributos ambientales junto con la pendiente, aspecto y área de influencia específica (47), (B) orientación: se considera únicamente aspectos estéticos, sin ningún tipo de restricción legal, teniendo además repercusiones en los atributos ambientales, (C) uso del suelo: para resolver los conflictos que surjan sobre la aceptación de integraciones de edificaciones no deseadas considerando el desarrollo actual de la tierra a partir de las bandas Landsat del modelo de elevación digital (MED), (D) visibilidad: con el objetivo de preservar la estética de las zonas habitadas a través de la evaluación de los puntos establecidos por los estudios de visibilidad, pero no basado en ninguna restricción legal (48).

Factores relativos a cuestiones ambientales: para el área de estudio seleccionada se analizaron los siguientes cuatro factores; (A) sensibilidad del ecosistema: lucha contra la contaminación potencial o la degradación de los medios naturales de interés o singularidad ecológica y/o estética, basándose en restricciones legales (49), (B) fuentes de agua: se considerarán a manantiales y/o pozos de agua subterránea mediante el cálculo de funciones de distancia euclidiana con el software ArcGIS 9.3, (C) aguas superficiales: lagos y ríos con flujo de agua continuo que sean potenciales receptores de residuos tratados o incluso sin tratar, (D) tipo de vegetación: se ha considerado tanto la singularidad ecológica de la vegetación boscosa, como la vegetación deforestada, y la distribución espacial de estas formaciones naturales. Para ellos se ha empleado el índice de diferencia normalizada de vegetación (IDNV).

Factores relativos a parámetros socio-económicos: para el área de estudio seleccionada se analizaron los siguientes cuatro factores; (A) infraestructura de acceso: se analizan las principales rutas de acceso para turistas, incluyendo las redes de transporte existentes como las autopistas, carreteras locales y ferrocarriles, (B) densidad de población: se considera una zona de influencia alrededor de las ciudades, pueblos y asentamientos humanos asociados a actividades económicas, (C) zonas residenciales: se consideran ciudades y pueblos que representan una alta concentración de actividad humana y su relación con la demanda de recursos de los alrededores, además de la presencia de los centros urbanos, (D) área de recurso turístico: se incluyen zonas turísticas, culturales y urbanas examinadas a través de diversos cálculos de distancia de cada zona y por las restricciones legales basadas en el uso del suelo y el tipo de cubierta.

\section{Restricciones:}

Las siguientes seis restricciones limitan el análisis de zonas geográficas concretas: (A) áreas de protección ambiental y ecosistemas sensibles según el reglamento de la comisión Europea por la naturaleza y políticas de biodiversidad (49), (B) acuíferos importantes, tales como manantiales y/o pozos de agua subterránea con alto riesgo de contaminación de las aguas subterráneas, (C) masas de agua superficial para evitar su contaminación, (D) tipos específicos de vegetación y usos del suelo con vegetación densa, (E) carreteras y vías férreas, siguiéndose los límites legales de distancia mínima, (F) áreas que prohíben la construcción de edificios comerciales a través de ordenanza regional de edificación.

Como última etapa del ADMC, se establecieron los valores relativos de peso y grado de importancia mediante PAJ a través del examen de varios indicadores con la ayuda de la matriz de comparación por pares (MCP) de cada criterio y sub-criterio. En último lugar, se utilizó el método de ponderación aditiva simple (PAS) para evaluar el índice de adecuación final, desde el área menos adecuada (1) a la más adecuada (10), para resolver el problema de criterios múltiples (37).

\subsection{Empleo de una interfaz web para la planificación espacial}

El potencial de disponer de una aplicación interactiva utilizando métodos ADMC será un punto de partida para el desarrollo de herramientas web sofisticadas. Con estas herramientas, cada usuario podrá participar de la manera más adecuada y expresar su experiencia individual, que podrá ser almacenada y utilizada para elaborar un mapa de conocimientos e intercambio de información.

La arquitectura de la herramienta web aprovecha la viabilidad que tienen estas aplicaciones para favorecer los procesos de toma de decisiones, identificar los modelos espaciales de las distintas percepciones y el intercambio de conocimientos para la integración de edificaciones y documentar el posible impacto sobre el turismo. Los usuarios podrán ir de forma consecutiva y fluida a través de la sección general, a la sección de ADMC, seguidamente a la sección de mapa de conocimiento y, por último, a la sección de cuestionario post-tarea. Se ha empleado el lenguaje de programación Smalltalk, lenguaje reflexivo orientado a objetos que emplea la arquitectura modelo-vista-controlador (MVC). Para iniciar la macro, los usuarios deben acceder a info.html y esta se desplegará a través del navegador web. Las cuatro secciones mostradas en la Figura 1 tienen la misma forma para todos los usuarios, independientemente si están o no autenticados.

\subsection{Medición de variables}

Los datos del estudio se recogieron a través de la página web, e-shift (http://www.e-shift.eu). A través de ella, los usuarios proporcionaron dos conjuntos de resultados, por un lado los rangos de ponderación del ADMC y por otro los resultados de la encuesta post-tarea. Un total de 258 participantes formaron parte del estudio. Estos participantes fueron categorizados en diferentes grupos en función de sus características socio-demográficas. El proceso de ponderación ADMC proporcionó un total de 243 respuestas, lo que supone un $94 \%$ del total de los usuarios de la página web. Por otro lado, la encuesta post-tarea, basada en la web, fue contestada por un total de 212 personas, lo que corresponde al $82 \%$ de los participantes.

\subsubsection{Clasificación de las ponderaciones del ADMC y cuestionario de encuesta}

La parte más significativa del proceso, y que supone un mayor esfuerzo, es el diseño, implementación y ejecución de las etapas necesarias para la determinación de las preferen- 


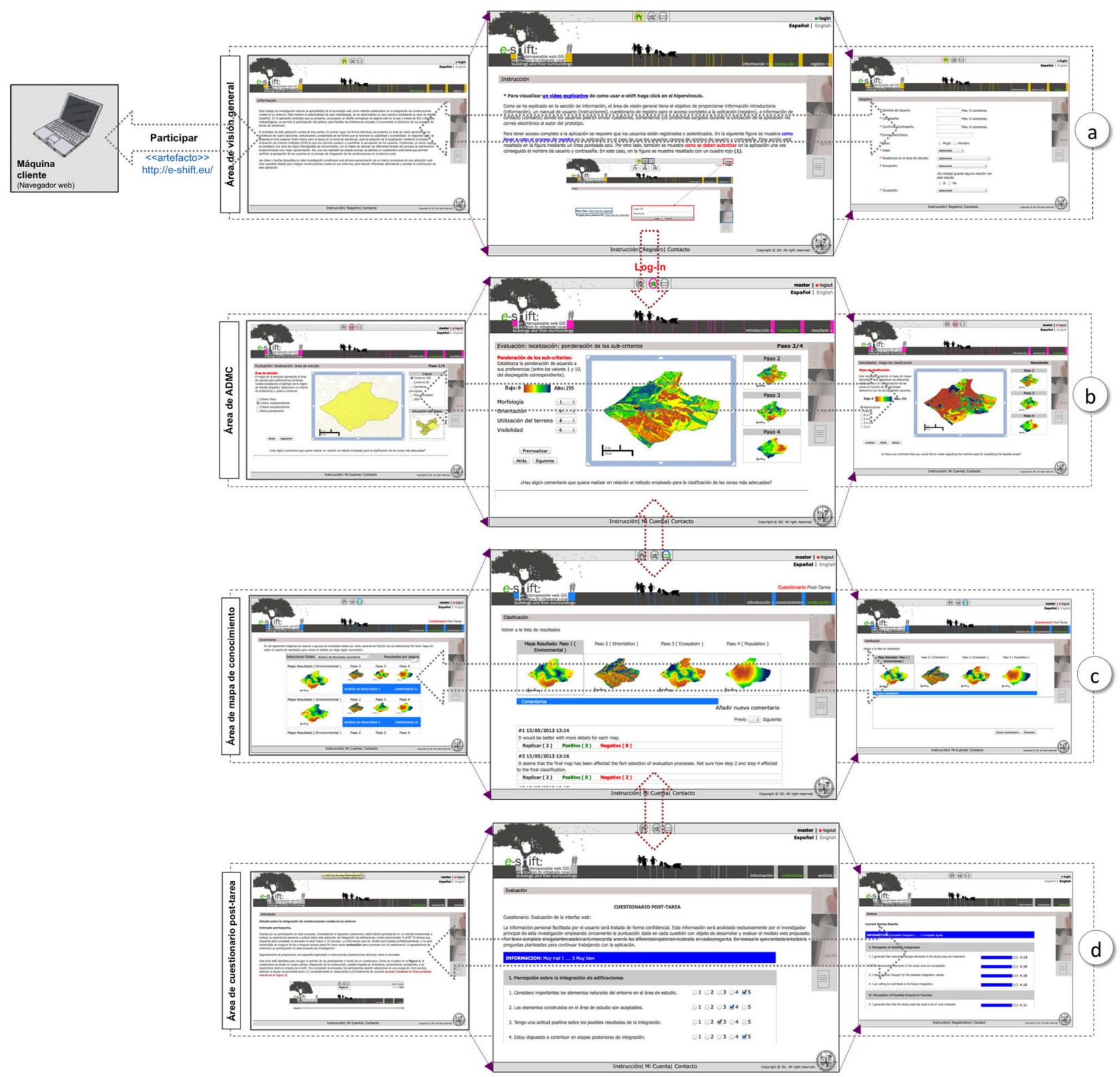

Figura 1. Proceso de implementación del flujo de trabajo de la página web e-shift.eu: a) secciones web con información general sobre la investigación, manual de usuario, información de contacto y formulario de registro; b) secciones dedicadas a las etapas de decisión espacial multi-criterio para el proceso espacial sugerido; c) secciones que muestra el mapa de conocimiento y las opciones de comunicación entre los participantes; d) cuestionario implementado en la web para establecer la percepción de los usuarios.

cias del ADMC y de la integración de las construcciones rurales en su entorno. En el proceso para determinar la ponderación del PAJ, los usuarios establecieron directamente su propio conjunto de ponderaciones a través de la página web. En concreto, los participantes pudieron seleccionar diferentes ponderaciones para cada uno de los tres criterios y cuatro sub-criterios establecidos. Dicha información constituye el conjunto de datos asignado por cada participante, que se almacenarán directamente en el sistema de gestión de bases de datos (SGBD). Después de la etapa de clasificación de ponderaciones a través de la página web, los participantes pueden completar el cuestionario de la encuesta post-tarea.

Durante el proceso de selección de emplazamientos para la integración de edificaciones rurales utilizando el ADMC, los usuarios pueden explorar el área de estudio mediante el examen de las diferentes capas del mapa, y posteriormente expresar sus preferencias a través de los tres criterios principales de decisión -a saber, criterios físicos, ambientales y socio-económicos- además el usuario tiene la opción de prorratear con una ponderación igualitaria los tres criterios, dependiendo de la preferencias de los usuarios, tal como se ha representado en la Figura 2a. Los usuarios deben iniciar sesión y seleccionar uno de los criterios de entre los tres que están disponibles. A este criterio seleccionado se le dará la máxima puntuación y los criterios restantes se ponderan con respecto a éste o bien el usuario puede seleccionar el mismo peso para los tres criterios con ayuda del método PAS. Esta técnica de simplificación ha sido descrita previamente por Easton y Malczewski basándose en el procedimiento de estimación de la relación (39) (50). En las siguientes páginas se muestran, como un único mapa, los tres criterios principales indicados anteriormente, donde los usuarios podrán pon- 


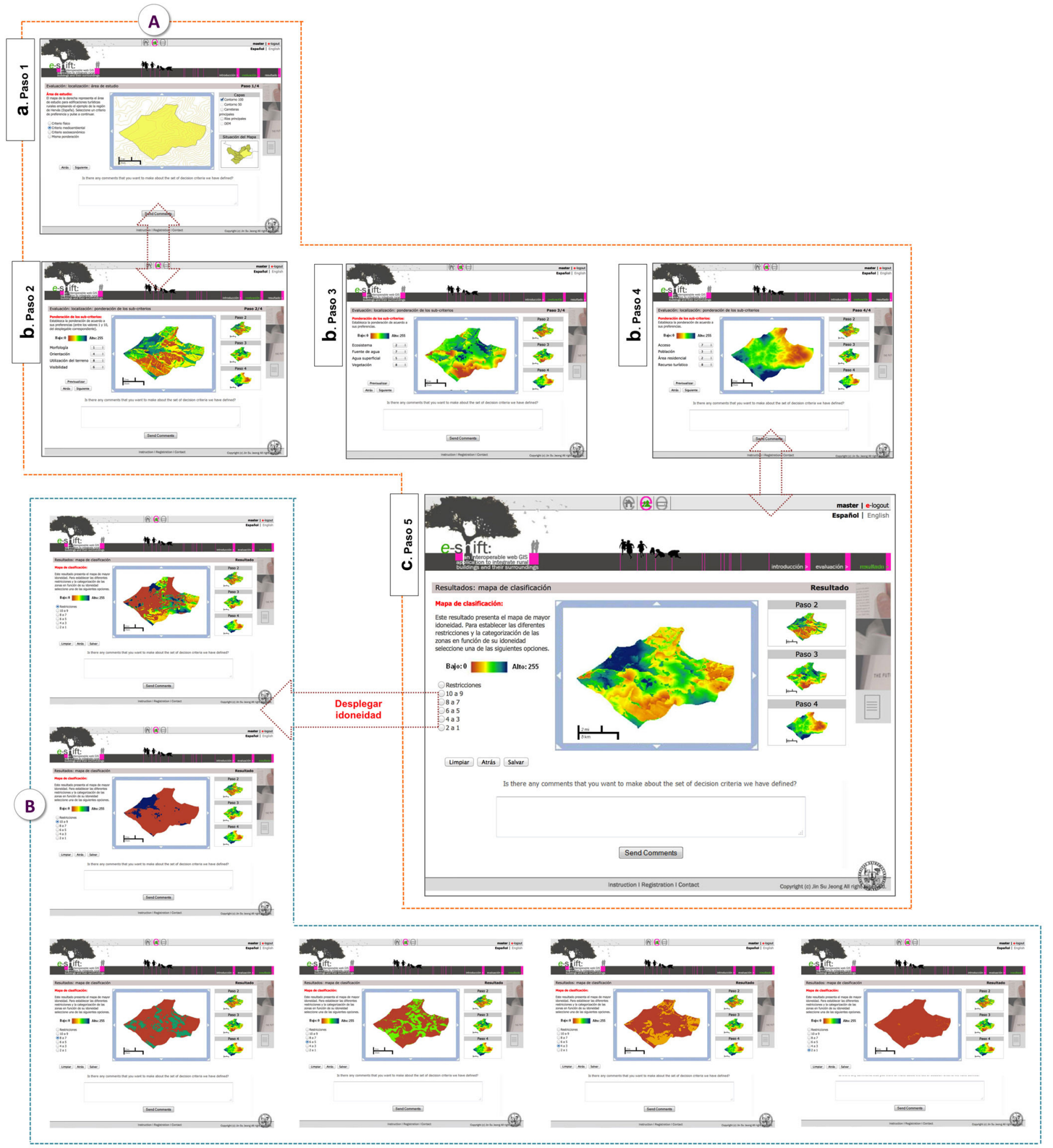

Figura 2. A) Sección de la web que muestran el proceso de selección del emplazamiento para la integración de construcciones rurales mediante el uso de ADMC basado en la selección de sub-criterios a partir de los tres criterios establecidos en el estudio.

B) Detalle del resultado obtenido al aplicar las restricciones y categorizaciones por parte de los usuarios.

derar cada uno de los sub-criterios asociados a cada criterio principal, mostrándose la importancia relativa del criterio de decisión (Figura 2b). Para establecer la importancia relativa de cada ponderación, se ha diseñado un menú desplegable que muestra un valor exacto, y donde se asigna el valor de 1 a la situación menos adecuada, y de 10 a la más adecuada a través del MCP, no siendo posible asignar la misma ponderación a diferentes sub-criterios de un mismo criterio. De este modo, los usuarios pueden así emplear sus opiniones para cuantificar la magnitud de los criterios y sub-criterios. Des- pués de evaluar todos los criterios de decisión y sub-criterios, en una página final se muestra el resultado de la clasificación. En esta página, los usuarios pueden ver diferentes mapas en función de las diferentes restricciones y la categorización de las zonas, en función de su idoneidad, mediante la selección de opción deseada en el menú correspondiente. Así, al seleccionar la restricción '10 a 9' el mapa final mostrará el área más adecuada en función de las ponderaciones dadas, y en la restricción '2 a 1' se mostrará el mapa indicando las zonas menos adecuadas (Figura 2c). En este punto, los usuarios son 
más conscientes de la tarea en la que están involucrados y, sin duda, están en mejores condiciones para juzgar los parámetros para seleccionar la localización (13).

Además, se ha diseñado un cuestionario, de la manera más clara posible mediante la aplicación de las normas más recientes, para establecer las preferencias individuales de un conjunto elevado de participantes (51). Con objeto de conseguir la mayor cantidad posible de respuestas, la recogida de datos se basa en una encuesta web interactiva que no sólo proporciona una mejor revelación cualitativa de preferencias, sino que también almacena directamente los resultados en un servidor de datos. Desde un punto de vista técnico, la página web ha sido diseñada para generar un entorno dinámico, y donde los resultados son transferidos y almacenados en la base de datos del servidor. Para el uso de esta aplicación no son necesarios plug-ins adicionales, ni se requiere ningún software especial por parte de los usuarios.

\subsubsection{Análisis de datos}

Se emplearon métodos de análisis cualitativos y cuantitativos para el análisis de datos. El análisis de contenido se centró en la recolección de datos cualitativos y cuantitativos sobre los distintos tipos del ADMC, el histórico de comentarios del ADMC, clasificaciones de conocimientos e historial de comentarios mediante la utilización de un esquema de codificación. Los resultados de la encuesta se expresaron como media \pm desviación estándar y se analizaron mediante un análisis de varianza (ANOVA) de una vía y la prueba Tuckey al nivel de significación $\mathrm{p} \leq \mathrm{0,1}$.

\section{RESULTADOS Y DISCUSIÓN}

Para fortalecer la interpretación de los resultados, se estableció y analizó el perfil socio-demográfico de los encuestados. Se realizó un análisis detallado tanto de la selección multicriterio y de los resultados de la encuesta proporcionado por los participantes, a fin de que la representatividad de la muestra pueda ser juzgada y tener una base estadística para los análisis posteriores.

\subsection{Perfil de los participantes}

La búsqueda de participantes se realizó a través de llamamientos mediante correo electrónico y de redes sociales. Las respuestas de los participantes fueron recogidas desde comienzo de enero de 2013 hasta finales de mayo de 2013. En total, 258 participantes, con diferentes orígenes y edades, visitaron la página web y participaron, posteriormente, en el proceso de clasificación ADMC, lo que arrojó un total de 243 valores de ponderación. Asimismo, 212 de los participantes completaron cuestionario post-tarea en línea. Debido a las características del área de estudio, los idiomas empleados en las encuestas fueron el inglés y el español. A partir de los datos obtenidos del estudio, se analizó el perfil socio-demográfico para interpretar los resultados alcanzados. Los antecedentes socio-demográficos de los encuestados se muestran en la Tabla 1.

Por otro lado, para reforzar la interpretación de los datos del estudio, los perfiles socio-demográficas fueron divididos y analizados dentro de cuatro grupos en función del grado de vinculación de los participantes con el área de estudio. Estos grupos fueron diseñados a través de la información recogi-
Tabla 1. Características socio-demográficas de los participantes de la encuesta $(n=212)$.

\begin{tabular}{|c|c|c|}
\hline $\begin{array}{l}\text { Cuestionario } \\
\text { de inscripción }\end{array}$ & Frecuencia & $\begin{array}{l}\text { Porcentaje } \\
\text { (\%) }\end{array}$ \\
\hline \multicolumn{3}{|l|}{ Sexo } \\
\hline Mujer & 99 & 46,7 \\
\hline Hombre & 113 & 53,3 \\
\hline \multicolumn{3}{|l|}{ Edad } \\
\hline Más de 65 & 8 & 3,8 \\
\hline 50 a 64 & 24 & 11,4 \\
\hline 35 a 49 & 81 & 37,5 \\
\hline 34 o más joven & 99 & 47,3 \\
\hline \multicolumn{3}{|l|}{ Residencia en el área de estudio } \\
\hline Residencia permanente & 39 & 18,6 \\
\hline Reside en la proximidad & 46 & 21,7 \\
\hline Segunda residencia & 16 & 7,4 \\
\hline No reside pero con fuertes intereses & 41 & 19,4 \\
\hline Sin ningún tipo de relación & 70 & 32,9 \\
\hline \multicolumn{3}{|l|}{ Educación } \\
\hline Educación elemental & 39 & 18,3 \\
\hline Educación secundaria & 37 & 17,6 \\
\hline Educación universitaria o superior & 136 & 64,1 \\
\hline \multicolumn{3}{|l|}{ Ocupación } \\
\hline $\begin{array}{l}\text { Guarda alguna relación con este } \\
\text { estudio }\end{array}$ & 110 & 51,7 \\
\hline Directivo/profesional/especialista & 5 & 4,9 \\
\hline Trabajo administrativo & 37 & 34,1 \\
\hline Obrero & 11 & 10,4 \\
\hline Otros & 57 & 50,6 \\
\hline $\begin{array}{l}\text { No guarda ninguna relación con } \\
\text { este estudio }\end{array}$ & 102 & 48,3 \\
\hline Directivo/profesional/especialista & 24 & 23,5 \\
\hline Trabajo administrativo & 34 & 33,3 \\
\hline Obrero & 15 & 14,4 \\
\hline Otros & 29 & 28,8 \\
\hline
\end{tabular}

da en la Tabla 1 considerando tanto aspectos sociales como emocionales. Los participantes del primer grupo corresponden a los que no tienen ningún tipo de relación con el área de estudio (grupo de vínculos débiles); los del segundo grupo poseen algún tipo de conexión social pero no emocional con el área, por ejemplo residentes ocasionales o vacacionales (grupo de socialmente vinculados); los del tercer grupo presentan fuertes vínculos con el área y por tanto cierto apego emocional con el lugar, es decir, viven permanentemente en el área de estudio o en sus cercanías (grupo de participantes altamente arraigados); y el último grupo lo integran aquellos que poseen un fuerte vínculo emocional pero no social, y que no residen en el área de estudio (grupo de participantes comprometidos con el lugar). 


\subsection{Percepción sobre la integración de edificaciones}

Los resultados empíricos del proceso de clasificación ADMC de los participantes se muestran en la Figura 3, según las distribuciones de criterios y sub-criterios, respectivamente. De entre las diferentes opciones de selección de criterios o igual ponderación entre ambos, los participantes optaron mayoritariamente por criterios medioambientales (65\%). En relación a los sub-criterios, el 55 \% seleccionó «la utilización del terreno» dentro de los criterios físicos, «tipo de vegetación» fue seleccionado por el $43 \%$ dentro de los criterios ambientales, y en relación a los sub-criterios socio-económicos la mayoría (46\%) optó por el «factor de acceso al sitio». En función del conjunto de resultados obtenidos, se pueden identificar las áreas más adecuadas, dentro de la zona de estudio, para la construcción de una nueva edificación turística rural.

La percepción que los participantes tuvieron sobre la integración de las construcciones, varía según su pertenencia a uno de los cuatro grupos descritos anteriormente. Como se muestra en la Tabla 2a, todos los participantes, independientemente del grupo de pertenencia, perciben de forma importante los elementos naturales del entorno (el valor medio combinado los cuatro grupos fue 4,13 -en la escala de cinco puntos-). Aun así el grupo de mayor arraigo presentó un resultado menos positivo comparado con los otros grupos (el valor medio fue de 3,90), siendo destacable la diferencia estadísticamente significativa de los valores otorgados por este grupo y los proporcionados por el grupo comprometido con el lugar (arraigos emocionales), como puede verse en los resultados del ANOVA (letras A, B y C). Los participantes con vínculos débiles presentaron una mayor predisposición a la integración, por tanto se registraron valoraciones más altas en relación a los elementos naturales del entorno. Con respecto a los resultados obtenidos en la percepción de los elementos construidos en el área de estudio fueron opuestos a los proporcionados en las cuestiones relativas a los elementos del paisaje. Así, en relación a la integración de edificaciones y su contribuciones futuras, casi la totalidad de los participantes y grupos aceptaron el proceso representación cartográfica otorgando resultados positivos, aunque se observó una pequeña diferencia entre las puntuaciones dadas por el «grupo de participantes altamente arraigados» y el «grupo comprometidos con el lugar» (los valores medios fueron 4,16 y 4,13, respectivamente). En general, los participantes con mayores lazos emocionales con el lugar, proporcionaron más respuestas negativas que aquellos solamente vinculados con lazos sociales. Por otro lado, este último grupo mostró una actitud más positiva que los participantes pertenecientes al grupo de individuos con vínculos débiles (indiferentes al área de estudio).

\subsection{Certificación de los posibles impactos en el turismo}

Las partes implicadas en el proceso de integración, mostraron grandes diferencias en la percepción de la importancia del impacto ecológico, social y económico, lo cual tuvo un efecto directo sobre el de preferencia de las alternativas de
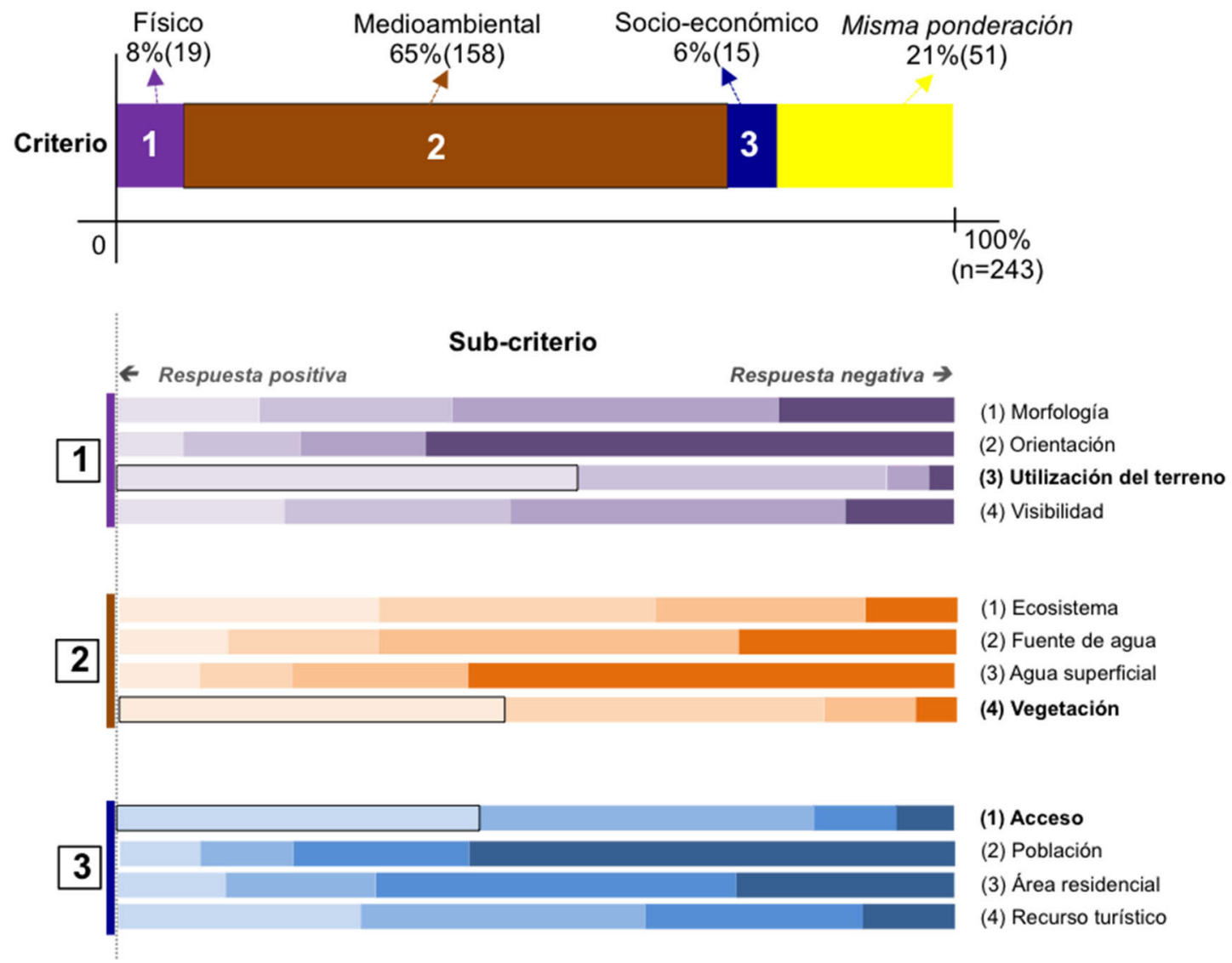

Figura 3. Resultados de la clasificación de las ponderaciones mediante ADMC de la distribución de criterios y sub-criterios dada por los participantes a través de la interfaz web $(n=243)$. En una misma barra, tonalidades menos intensas denotan respuestas positivas, y tonalidades más intensas indican respuestas negativas. 
Tabla 2. Resultados de la encuesta realizada a los participantes y comparación de valores medios dentro de cuatro grupos (nivel de significación $\mathrm{p} \leq \mathrm{0}, 1$ ).

\begin{tabular}{|c|c|c|c|c|c|c|}
\hline \multirow{2}{*}{\multicolumn{2}{|c|}{ Cuestionarios }} & \multicolumn{5}{|c|}{ Puntuación media (Escala de 5 puntos, mínimo $1 \ldots 5$ máximo) } \\
\hline & & \multirow{2}{*}{$\begin{array}{l}\text { Vínculos } \\
\text { débiles }\end{array}$} & \multirow{2}{*}{$\begin{array}{c}\text { Social- } \\
\text { mente } \\
\text { vinculados }\end{array}$} & \multirow{2}{*}{$\begin{array}{l}\text { Altamente } \\
\text { arraigados }\end{array}$} & \multirow{2}{*}{$\begin{array}{l}\text { Comprome- } \\
\text { tidos } \\
\text { con el lugar }\end{array}$} & \multirow{2}{*}{ Todos } \\
\hline \multirow{5}{*}{ a. } & Percepción sobre la integración de edificaciones & & & & & \\
\hline & $\begin{array}{l}\text { C1. Considero importantes los elementos naturales } \\
\text { del entorno en el área de estudio. }\end{array}$ & $4,25 \mathrm{~A}, \mathrm{~B}$ & $4,04 \mathrm{~B}, \mathrm{C}$ & $3,90 \mathrm{C}$ & $4,34 \mathrm{~A}$ & 4,13 \\
\hline & $\begin{array}{l}\text { C2. Los elementos construidos en el área de estudio } \\
\text { son aceptables. }\end{array}$ & $3,96 \mathrm{~A}, \mathrm{~B}$ & $4,15 \mathrm{~A}, \mathrm{~B}$ & $4,31 \mathrm{~A}$ & $3,83 \mathrm{C}$ & 4,06 \\
\hline & $\begin{array}{l}\text { C3. Tengo una actitud positiva sobre los posibles } \\
\text { resultados de la integración. }\end{array}$ & $4,09 \mathrm{~A}, \mathrm{~B}$ & 4,19A,B & $4,33 \mathrm{~A}$ & $4,02 B$ & 4,16 \\
\hline & $\begin{array}{l}\text { Q4. Estoy dispuesto a contribuir en etapas posterio- } \\
\text { res de integración. }\end{array}$ & $4,05 \mathrm{~A}, \mathrm{~B}$ & $4,22 \mathrm{~A}, \mathrm{~B}$ & $4,28 \mathrm{~A}$ & $3,95 \mathrm{~B}$ & 4,13 \\
\hline \multirow{5}{*}{ b. } & \multicolumn{6}{|l|}{ Percepción del posible impacto en el turismo } \\
\hline & $\begin{array}{l}\text { C5. Considero que el área de estudio tiene muchas } \\
\text { características rurales. }\end{array}$ & $4,03 \mathrm{~B}$ & $4,06 \mathrm{~A}, \mathrm{~B}$ & $3,90 \mathrm{~B}$ & $4,33 \mathrm{~A}$ & 4,08 \\
\hline & $\begin{array}{l}\text { C6. La preservación de las características rurales } \\
\text { son de gran importancia para el turismo. }\end{array}$ & $4,22 \mathrm{~A}$ & $3,93 \mathrm{~B}$ & $3,85 \mathrm{~B}$ & $4,36 \mathrm{~A}$ & 4,09 \\
\hline & $\begin{array}{l}\text { C7. Estoy convencido que los gobiernos locales } \\
\text { apoyan las actividades turísticas. }\end{array}$ & $4,18 \mathrm{~A}$ & $3,89 \mathrm{~B}$ & $3,96 \mathrm{~A}, \mathrm{~B}$ & 4,17A,B & 4,05 \\
\hline & $\begin{array}{l}\text { C8. Este modelo muestra de forma adecuada donde } \\
\text { deben producirse desarrollos turísticos. }\end{array}$ & 4,05 & 4,24 & 4,24 & 3,97 & 4,12 \\
\hline \multirow{5}{*}{ c. } & \multicolumn{6}{|l|}{ Percepción sobre el conocimiento compartido } \\
\hline & $\begin{array}{l}\text { C9. El área de intercambio de comentarios/cono- } \\
\text { cimientos en esta aplicación es importante para el } \\
\text { proceso de planificación. }\end{array}$ & $4,32 \mathrm{~A}$ & $4,11 \mathrm{~A}, \mathrm{~B}$ & $3,96 \mathrm{~B}$ & $4,28 \mathrm{~A}$ & 4,17 \\
\hline & $\begin{array}{l}\text { C10. Considero que los mapas de conocimiento son } \\
\text { bastantes útiles y efectivos para compartir y reutili- } \\
\text { zar las experiencias de otros usuarios. }\end{array}$ & $4,29 \mathrm{~A}$ & 4,09A,B & $4, \mathrm{OoB}$ & $4,24 \mathrm{~A}, \mathrm{~B}$ & 4,16 \\
\hline & $\begin{array}{l}\text { C11. He comprobado la clasificación realizada por } \\
\text { otros participantes al menos una vez cuando he } \\
\text { trabajado con esta aplicación. }\end{array}$ & 4,26 & 4,07 & 3,97 & 4,26 & 4,14 \\
\hline & $\begin{array}{l}\text { C12. Tener un acceso directo al mapa de conoci- } \\
\text { miento es de gran utilidad. }\end{array}$ & $4,33 \mathrm{~A}$ & $4,11 \mathrm{~A}, \mathrm{~B}$ & $4,03 \mathrm{~B}$ & $4,24 \mathrm{~A}, \mathrm{~B}$ & 4,18 \\
\hline \multirow{5}{*}{ d. } & \multicolumn{6}{|l|}{ Percepción sobre la usabilidad de la página web } \\
\hline & $\begin{array}{l}\text { C13. Creo que el sistema es auto-explicativo, lo } \\
\text { que indica que el navegador web proporciona una } \\
\text { interfaz de usuario comprensible. }\end{array}$ & 4,28 & 4,11 & 4,01 & 4,29 & 4,17 \\
\hline & $\begin{array}{l}\text { C14. La interfaz muestra menús descriptores, } \\
\text { submenús, formatos de imágenes y tablas de forma } \\
\text { adecuada. }\end{array}$ & 4,32 & 4,11 & 4,00 & 4,29 & 4,28 \\
\hline & $\begin{array}{l}\text { C15. El sistema no necesita software adicional o } \\
\text { plug-ins para ser ejecutado de forma apropiada. }\end{array}$ & 4,26 & 4,09 & 4,00 & 4,31 & 4,27 \\
\hline & $\begin{array}{l}\text { C16. El sistema puede detectar mensajes de error } \\
\text { cuando la información introducida no posee el for- } \\
\text { mato adecuado o no es válida para el sistema. }\end{array}$ & 4,33 & 4,29 & 4,15 & 4,28 & 4,24 \\
\hline
\end{tabular}

Nota: Diferentes letras en la misma fila indica la existencia de diferencias significativas entre los diferentes grupos para un nivel de significación de o,1. Mismas letras en la misma fila indica la no existencia de diferencias significativas entre los valores para ese nivel de significación.

planificación. A la pregunta sobre el posible impacto en el turismo (Tabla 2b) los usuarios de la aplicación web opinaron que el área de estudio tenía un gran carácter rural (el valor promediado de los cuatro grupos fue de 4,08), y que la preservación de este carácter rural constituía un aspecto esencial para el turismo (la media de todos los valores fue de 4,09). Estas respuestas muestran una coherencia con las actitudes de los encuestados hacia el mantenimiento de carácter rural, y ponen de manifiesto que el carácter ru- ral del área debe ser considerado. De forma específica, los participantes del estudio con un alto grado de apego emocional, proporcionaron respuestas más positivas que los participantes con un mayor arraigo social, concretamente el grupo de participantes comprometidos con el lugar, otorgó una puntuación de 4,33 y 4,36 respectivamente, mientras que los valores de puntación dados por el grupo de participantes altamente arraigados fue de 3,90 y 3,85 respectivamente. 
En el cuestionario se incorporaron dos preguntas relativas a la actitud de los participantes en cuestiones de planificación, incluyendo el apoyo general de la planificación, la protección de los recursos y la planificación de estrategias específicas. En primer lugar, se preguntó a los encuestados si consideraban, que de forma general, los gobiernos locales apoyaban a las actividades turísticas de la zona de estudio. De forma general, la mayoría de los participantes aportaron respuestas afirmativas, sin embargo en función del arraigo en términos sociales, con la zona de estudio los resultados difieren significativamente (denotado con letras A y B en la Tabla 2b). Tan sólo un $22 \%$ de los encuestados calificaron el apoyo de los gobiernos locales como bajo (puntuaciones entre 1 y 3 ), mientras que un $28 \%$ consideraron el apoyo gubernamental como «muy importante» (puntuación 5). Otra de las preguntas recogidas en la encuesta profundizaba sobre la actitud de los participantes hacia el mapa de idoneidad, presentado en el modelo, sobre donde debería producirse un nuevo desarrollo turístico. Los resultados mostraron que la mayoría de los participantes estuvieron de acuerdo con el mapa final sobre la ubicación adecuada (la puntuación promediada de todos los participantes para esta pregunta fue de 4,12), sin que existieran diferencias significativas entre los participantes pertenecientes a los diferentes grupos.

\subsection{Evaluación del intercambio de conocimientos}

Para evaluar el proceso de intercambio de conocimiento a través la aplicación web y su resultado, se analizaron la frecuencia de los resultados de la clasificación de los usuarios, además de llevar a cabo una encuesta on-line. Como primer paso, se obtiene un archivo con los resultados de clasificación de todos los usuarios, y se elabora un mapa de conocimiento del proceso de clasificación ADMC, a partir de él es posible comprobar las acciones de intercambio mediante el acceso a los datos (Figura 1c). Así, el mapa del conocimiento es el recurso definitivo de esta aplicación, y es adecuado para la documentación, el intercambio y la reutilización de información por parte de los usuarios. Todos los comentarios entre los usuarios se guardan en una base de datos como un registro de intercambio de conocimiento efectivo personal. Este podrá ser transferido y aplicado para otros procesos por otros usuarios. Por ejemplo, los usuarios podrán leer y evaluar las contribuciones realizadas previamente por otros usuarios y así tener otras perspectivas sobre la idoneidad de las ubicaciones, por lo que incluso podrán evaluar y quizá revisar sus propias clasificaciones.

A partir de los resultados obtenidos en la encuesta (Tabla 2c) se pudo comprobar que en general, los usuarios consideraron importante la interfaz de comunicación en el proceso de planificación (la puntuación promedia dada fue de 4,17). Por tanto, el mapa del conocimiento fue una etapa útil y efectiva en el intercambio y la reutilización de las opiniones de los participantes, las cuales fueron archivadas en la base de datos del servidor (la puntuación promedia dada fue de 4,16). En estas dos preguntas, el «grupo de participantes altamente arraigados» otorgó una respuesta menos positiva que la dada por los otros. En relación a las preguntas que comprobaban la utilización real del mapa de conocimiento, los usuarios que opinaron, una vez que habían consultado las clasificaciones realizadas por otros participantes, indicaron que tras realizar esta consulta habían cambiado su opinión, por lo que realizaron una nueva clasificación para obtener resultados diferentes. Los usuarios consultaron, al menos una vez, la clasificación realizada por otros participantes durante la utilización de la aplicación web (la puntuación promedia dada fue de 4,14). Además indicaron que el acceso directo al mapa de conocimiento fue de gran utilidad, aunque el "grupo de participantes altamente arraigados» proporcionaron una puntuación algo menor.

\subsection{Evaluación de la usabilidad de la página web}

Todo software presenta algún tipo de limitación; la evaluación de estas limitaciones es una forma apropiada de medir su funcionalidad. Así, un desarrollador de software debe analizar las limitaciones del programa a la luz de las restricciones de su funcionamiento, y resolver los problemas más habituales - por lo que se evidencia la necesidad de que el desarrollador debe estar altamente calificado (52) (53). El proceso de evaluación debe incluir una revisión completa del funcionamiento del programa en el hardware del equipo, y esta funcionalidad debe probarse en múltiples equipos. Por tanto, la evaluación de la usabilidad del programa involucra la comprobación de los diferentes componentes del software para ver si están conformes con la guía de evaluación de software de ingeniería.

Antes de publicar la página web, se verificó que el sistema podía llevar a cabo todas sus funciones de forma adecuada. Seguidamente para comprobar la usabilidad de la web, se evaluaron los resultados de la encuesta on-line realizada por los usuarios (212 participantes completaron el cuestionario). A partir de estos datos (Tabla 2d) se obtuvieron los siguientes resultados: los usuarios encontraron que el sistema era autoexplicativo, en el sentido de que su navegador web les proporcionó una página fácilmente entendible (puntuación promedia de 4,17); la mayoría de los participantes respondieron que la web empleaba gráficos y métodos de interfaz adecuados tales como menús, submenús, los formatos de imagen y tablas (puntuación promedia de 4,28). Además, los datos mostraron que el sistema no requería, para un funcionamiento adecuado, software adicional o plug-ins (puntuación promedia de 4,27). Finalmente, también se indica que el sistema envía mensajes de error cuando el usuario introduce datos con un formato no adecuado o información no válida, para su corrección antes de continuar con el proceso (puntuación promedia de 4,24). A diferencia de lo observado anteriormente, en esta serie de preguntas no hubo diferencias estadísticamente significativas entre los diferentes grupos de usuarios.

\section{CONCLUSIONES}

En este trabajo se presenta un entorno web de participación mutua, denominado e-shift, para la ordenación del territorio y se describe su aplicabilidad para apoyar la toma de decisiones en el proceso de integración de construcciones turísticas rurales en su entorno. En este trabajo se ha implementado y probado una aproximación a los procesos de agrupamiento PAJ/PSA para generar una amplia gama de alternativas de decisión basadas en esta página web. Mediante el análisis de la selección multi-criterio llevada a cabo por los participantes, se ha comprobado que los usuarios muestran las siguientes preferencias para los diferentes criterios y sub-criterios: el criterio medioambiental fue el más frecuentemente seleccionado (65\%), los sub-criterios utilización del suelo (55\%), tipo de vegetación ( $43 \%$ ) y acceso al sitio (46 \%) fueron los más seleccionados dentro de los criterios físicos, ambientales y socio-económicos respectivamente. Así, mediante la eva- 
luación cualitativa y cuantitativa del conjunto de datos obtenidos mediante el análisis de la información facilitada por los usuarios en la encuesta, ha sido posible clarificar las opiniones de los participantes, y hubo un consenso positivo en relación a la hipótesis de investigación propuesta (la puntuación promedia de todos los grupos superior a 4).

Considerando los datos de la Tabla 2, el análisis de factores de los cuatro grupos de participantes, en relación al área de estudio, reveló que los grupos se diferenciaban por sus propias características y por su apego con la zona de estudio en términos sociales y emocionales. Es interesante destacar, de acuerdo con los resultados del ANOVA, que el "grupo de participantes altamente arraigados» presentó mayores diferencias con el resto de grupos en algunos de los estudios de percepción realizados. De forma particular, en la cuestión relativa a la integración de edificios, este grupo mostró más diferencias que los otros, proporcionando respuestas más positivas a esta categoría que el resto. Este grupo tam- bién mostró una actitud más positiva hacia la integración de construcciones y su impacto en el turismo en comparación con el «grupo de participantes comprometidos con el lugar», que tuvieron una actitud más negativa a estas mismas cuestiones. Además se evaluaron las diferencias existentes entre los grupos en términos sociales y emocionales, en particular, en las categorías de intercambio de conocimientos y pruebas de usabilidad. Según los resultados del ANOVA, los grupos usuarios sin o con poco arraigo social aportaron respuestas más positivas. Estos resultados muestran que la aplicación es espacial, simple y flexible, además permite facilitar las diferentes implementaciones metodológicas derivadas de las alternativas generadas en el proceso de toma de decisiones y el intercambio de conocimiento entre los participantes.

\section{AGRADECIMIENTOS}

Jin Su Jeong agradece la financiación de la Universidad de Extremadura (Contrato puente para becarios predoctorales).

\section{REFERENCIAS}

(1) Jeong, J.S. (2014). A collaborative planning approach using multi-criteria spatial decision analysis to integrate rural buildings into a landscape in GIS-enabled web environment (Ph.D. Thesis). University of Extremadura, Spain.

(2) Jeong, J.S., García-Moruno, L., Hernández-Blanco, J. (2013). A site planning approach for rural buildings into a landscape using a spatial multi-criteria decision analysis methodology. Land Use Policy, 32: 108-118, doi: http://dx.doi. org/10.1016/j.landusepol.2012.09.018.

(3) Jaraíz, F.J., Mora, J., Gutiérrez, J.A., Jeong, J.S. (2013). Comparison of regional planning strategies: countywide general plans in USA and territorial plans in Spain. Land Use Policy, 30(1): 758-773, doi: http://dx.doi.org/10.1016/j.landusepol.2012.06.001.

(4) Mennella, V. (1997). Qualità dell'ambiente e sviluppo delle aree rurali. Perugia : Genio Rurale, Edagricole.

(5) De Kadt, E. (1979). Tourism: passport to development? Oxford: Oxford University Press.

(6) Pigram, J. (1980). Environmental implications of tourism development. Annals of Tourism Research, 7(4): 554-583, doi: http://dx.doi.org/10.1016/0160-7383(80)90049-3.

(7) Díaz-Martín, A. M., Vázquez-Casielles, R. (1998). La calidad de servicio percibida por los clientes y por las empresas de turismo rural. Revista Española de Investigación de Marketing, 2(1): 31-54.

(8) Gascón-Linares, M.A. (1993). Turismo rural en España. Madrid: Secretaría General Técnica, Ministerio de Agricultura, Pesca y Alimentación.

(9) World Tourism Organisation (WTO). (2005). WTO World Tourism Barometer, vol. 3, nº 2. http://www.e-unwto.org/ toc/wtobarometeresp/3/2.

(10) Council of the European Union. (2001). Council resolution on architectural quality in urban and rural environments (2001/C 73/04). Official Journal of the European Communities, C73, 6/3/2001.

(11) De Vriesa, S., de Grootb, M., Boersb, J. (2012). Eyesores in sight: quantifying the impact of man-made elements on the scenic beauty of Dutch landscapes. Landscape and Urban Planning, 105(1-2): 118-127, doi: http://dx.doi.org/10.1016/j. landurbplan.2011.12.005.

(12) García-Moruno, L., Hernández-Blanco, J., Ayuga, F. (2003). Analysis of the exterior colour of agroindustrial buildings: a computer aided approach to landscape integration. Journal of Environmental Management, 69(1): 93-104, doi: http:// dx.doi.org/10.1016/So301-4797(03)00121-X.

(13) Jeong, J.S., García-Moruno, L., Hernández-Blanco, J. (2012). Integrating buildings into a rural landscape using a multicriteria spatial decision analysis in GIS-enabled web environment. Biosystems Engineering, 112(2): 82-92, doi: http:// dx.doi.org/10.1016/j.biosystemseng.2012.03.002.

(14) Tassinari, P., Torreggiani, D., Paolinelli, G., Benni, S. (2007). Rural buildings and their integration in landscape management. Agricultural Engineering International: the CIGR Ejournal, Vol. IX, Manuscript LW 07020.

(15) Bell, S. (1995). Elements of visual design in the landscape. London: E\&FN Spon.

(16) Tandy, C. (1979). Industria y paisaje. Madrid: Ed Leonard Hill Books.

(17) Craig, W.J., Harris, T.M., Weiner, D. (2002). Community participation and geographic information system. London \& New York: Taylor \& Francis.

(18) Olson, M. (1965). The logic of collective action; public goods and the theory of groups. Cambridge, MA: Harvard University Press.

(19) Jones, B. (1990). Neighborhood planning: A guide for citizens and planners. Chicago and Washington, D.C: Planners Press, American Planning Association.

(20) Fountas, S., Wulfsohn, D., Blackmore, B.S., Jacobsen, H.L., Pederson, S.M. (2006). A model of decision-making and information flows for information-intensive agriculture. Agricultural Systems, 87 (2): 192-210, doi: http://dx.doi. org/10.1016/j.agsy.2004.12.003. 
(21) Böhme, K., Schön, P. (2006). From Leipzig to Leipzig: territorial research delivers evidence for the new territorial agenda of the European Union. disP, 165(2): 61-70.

(22) Domingo-Santos, J.M., Fernández de Villarán, R., Rapp-Arrarás, I., Corral-Pazos de Provens, E. (2011). The visual exposure in forest and rural landscapes: an algorithm and a GIS tool. Landscape and Urban Planning, 101(1): 52-58, doi: http://dx.doi.org/10.1016/j.landurbplan.2010.11.018.

(23) García-Moruno, L., Hernández-Blanco, J., Ayuga-Téllez, F., García-Navarro, J. (1998). Localización de las construcciones en el ámbito rural ante la protección del paisaje. Informes de la Construcción, 50(458): 61-70, doi: http://dx.doi. org/10.3989/ic.1998.v50.i458.879.

(24) Hermann, S., Osinski, E. (1999). Planning sustainable land use in rural areas at different spatial levels using GIS and modelling tools. Landscape and Urban Planning, 46(1-3): 93-101, doi: http://dx.doi.org/10.1016/So1692046(99)00050-X.

(25) Hernández-Blanco, J., García-Moruno, L., Ayuga, F. (2004). Assessment of the visual impact made on the landscape by new buildings: a methodology for site selection. Landscape and Urban Planning, 68(1): 15-28, doi: http://dx.doi. org/10.1016/So169-2046(03)00116-6.

(26) Jankowski, P., Nyerges, T., Smith, A., Moore, T.J., Horvath, E. (1997). Spatial group choice: a SDSS tool for collaborative spatial decision-making. International Journal of Geographical Information Systems, 11(6): 577-602, doi: http:// dx.doi.org/10.1080/136588197242202.

(27) Bishop, I.D., Stock, C. (2010). Using collaborative virtual environments to plan wind energy installations. Renewable Energy, 35(10): 2348-2355, doi: http://dx.doi.org/10.1016/j.renene.2010.04.003.

(28) Bishop I.D., Stock, C., Williams, K. (2009). Using virtual environments and agent models in multi-criteria decision making. Land Use Policy, 26(1): 87-94, doi: http://dx.doi.org/10.1016/j.landusepol.2008.01.010.

(29) Ruiz, M.C., Fernández, I. (2009). Environmental assessment in construction using a spatial decision Support system. Automation in Construction, 18(8): 1135-1143, doi: http://dx.doi.org/10.1016/j.autcon.2009.07.005.

(30) Voss, A., Denisovich, I., Gatalsky, P., Gavouchidis, K., Klotz, A., Roeder, S., Voss, H. (2004). Evolution of a participatory GIS. Computers, Environment and Urban Systems, 28(6): 635-636, doi: http://dx.doi.org/10.1016/j.compenvurbsys.2003.12.003.

(31) Batty, M., Dodge, M., Jiang, B., Smith, A. (1998). GIS and urban design (Working Paper, No. 3). London: Centre for Advanced Spatial Analysis, University College London.

(32) Jeong, J.S., García-Moruno, L., Hernández-Blanco, J. (2014). Un modelo WEB para la asistencia en la toma de decisiones en la integración de las construcciones rurales mediante planificación espacial multi-criterio. Informes de la Construcción, 66(533): eo04, doi: http://dx.doi.org/10.3989/ic.13.001.

(33) Kingston, R., Carver, S., Evans, A., Turton, I. (2000). Web-based public participation geographical information systems: an aid to local environmental decision-making. Computers, Environment and Urban Systems, 24 (2): 109-125, doi: http://dx.doi.org/10.1016/So198-9715(99)00049-6.

(34) Al-Kodmany, K. (2001). Supporting image ability on the World Wide Web: Lynch's five elements of the city in community planning. Environment and Planning B: Planning and Design, 28(6): 805-832, doi: http://dx.doi.org/10.1068/ b2746t.

(35) Voinov, A., Bousquet, F. (2010). Modeling with stakeholders. Journal of Environmental Modeling \& Science, 25(11): 1268-1281, doi: http://dx.doi.org/10.1016/j.envsoft.2010.03.007.

(36) Armengou, J., Aguado, A., Ormazábal, G. (2012). Sistema integrado para toma de decisiones en el diseño de estructuras de hormigón. Informes de la Construcción, 64(527): 391-400, doi: http://dx.doi.org/10.3989/ic.11.019.

(37) Hwang, C.L., Yoon, K.L. (1981). Multiple attribute decision making: methods and applications, vol. 186. New York: Springer-Verlag. Doi: http://dx.doi.org/10.1007/978-3-642-48318-9.

(38) Keefer, D.L., Kirkwood, C.W., Corner, J.L. (2004). Perspective on decision analysis applications, 1990-2001. Decision Analysis, 1(1): 4-22, doi: http://dx.doi.org/10.1287/deca.1030.0004.

(39) Malczewski, J. (1999). GIS and multicriteria decision analysis. New York : John Wiley \& Sons.

(40) Roy, B. (1996). Multicriteria methodology for decision aiding. Dordrecht: Kluwer Academic Publishers.

(41) Gemitzi, A., Petalas, C., Tsihrintzis, V., Pisinaras, V. (2006). Assessment of groundwater vulnerability to pollution: a combination of GIS, fuzzy logic and decision making techniques. Environmental Geology, 49(5): 653-673, doi: http:// dx.doi.org/10.1007/s00254-006-0359-1.

(42) Saaty, T.L. (1977). A scaling method for priorities in hierarchical structures. Journal of Mathematical Psychology, 15(3): 234-281, doi: http://dx.doi.org/10.1016/0022-2496(77)90033-5.

(43) Saaty, T.L. (1996). The analytic hierarchy process. New York: McGraw-Hill.

(44) Jeong, J.S., García-Moruno, L., Hernández-Blanco, J., Jaraíz-Cabanillas, F.J. (2014). An operational method to supporting siting decisions for sustainable rural second home planning in ecotourism sites. Land Use Policy, 41: 550-560, doi: http://dx.doi.org/10.1016/j.landusepol.2014.04.012.

(45) Hernández, J., García, L., Montero, M., Sánchez, A. López, S. (2007). Determinación de impactos producidos en los humedales de Extremadura para su defensa y protección ambiental. Plasencia, Spain: Universidad de Extremadura.

(46) LESOTEX. (2001). Guía práctica de aplicación de la Ley 15/2001 del suelo y ordenación territorial de Extremadura. http://fomento.gobex.es/fomento/live/consejeria/legislacion/NORMATIVAUrbanismo/guia.pdf.

(47) Gallant, J.C., Wilson, J.P. (2000). Primary topographic attributes. En Wilson, J.P., Gallant, J.C. (Eds.), Terrain analysis principles and applications (pp. 51-85). New York: John Wiley \& Sons, Inc.

(48) Hernández-Blanco, J., García-Moruno, L., Morán, J., Juan, A., Ayuga, F. (2003). Estimating visual perception of rural landscapes using GIS: the influence of vegetation. Journal of Food, Agriculture and Environment, 1(1): 139-141. 
(49) NATURA. (2000). European commission environment for nature \& biodiversity policy. http://ec.europa.eu/environment/nature/natura20oo/index_en.htm.

(50) Easton, A. (1973). Complex managerial decision involving multiple objectives. New York: John Wiley \& Sons.

(51) Reips, U.D. (2002). Standards for internet-based experimenting. Journal of Experimental Psychology, 49(4): $243-256$.

(52) Mustajoki, J., Hämäläinen, R.P., Marttunen, M. (2004). Participatory multicriteria decision analysis with Web-HIPRE: a case of lake regulation policy. Environmental Modelling \& Software, 19(6): 537-547, doi: http://dx.doi.org/10.1016/j. envsoft.2003.07.002.

(53) Hand, T. (1989). Guide for evaluation engineering software. New York: The American Society of Civil Engineers. 